\title{
STM Imaging of Vortices in FIB-Sculptured Mesoscopic Superconductors
}

\author{
G. Karapetrov,* J. Fedor, ${ }^{* *}$ M. Iavarone,* M.T Marshall***, R. Divan****, W.K. Kwok* \\ * Materials Science Division, Argonne National Laboratory, Argonne, IL 60439 \\ ** also at Institute of Electrical Engineering, Slovak Academy of Sciences, Dubravska Cesta 9, \\ 84104 Bratislava, Slovak Republic \\ *** Center for Microanalysis of Materials, University of Illinois - Urbana-Champaign, Urbana, IL \\ 81801 \\ **** Center for Nanoscale Materials, Argonne National Laboratory, Argonne, IL 60439
}

One of the main criteria determining the usefulness of superconductors is the amount of critical current that the material can carry without dissipation. Vortex movement or creep is the main source of power loss in both low-temperature and high-temperature superconductors (HTS). Reduction of the vortex creep at high magnetic fields and high temperatures can be achieved by inclusion of nonsuperconducting centers with sizes on the order of the vortex core. The pinning force exhibited by the pinning centers on the Abrikosov vortices can be formidable and it can lead to critical current enhancement that is several orders of magnitude.

Scanning tunneling microscopy (STM) measures the local density of states on the surface of the superconductor to resolve individual vortices. So far only limited success in STM vortex imaging has been accomplished in materials other than pristine single crystals due to the extreme requirements on surface quality for STM [1,2]. We present novel STM vortex images in superconducting-normal metal heterostructures at magnetic fields that are up to 100 times of the matching field of the system and close to real application conditions. The described fabrication method using focused ion beam (FIB) fabrication opens possibilities to study vortex pinning and vortex dynamics in the presence of controlled nano-size pinning centers.

$\mathrm{NbSe}_{2}$ single crystals were grown in evacuated quartz ampoules by an iodine vapour transport method [3]. Flat samples with sizes of up to $10 \times 10 \times 0.1 \mathrm{~mm}^{3}$ were characterized by Xray, transport, and magnetization measurements. SQUID magnetization measurements show typical superconducting transitions of $7.2 \mathrm{~K}$ with transition width of $10 \mathrm{mK}$. As-grown single crystals were cleaved to expose a clean flat surface. Using a focused beam of gallium ions (FEI DB-235) we etched a set of $1 \mu \mathrm{m}$ deep parallel grooves spaced equidistantly $0.5 \mu \mathrm{m}$ apart (Fig.1). A second set of identical grooves, orthogonal to the original one, was inscribed in the same area producing an array of single crystal pillars (Fig.1e). Although the modulated surface looks smooth under high resolution scanning electron micrograph (SEM) we were not able to obtain superconductor-insulator-normal metal tunnelling spectra by STM. It is most likely that the Ga ions and the debris from ion milling rapidly contaminate the STM tip.

To resolve the above problem we proceeded with microfabrication by electroplating two micrometers of gold on the patterned surface. We used a Techni-gold 25E (Technic Inc.) electroplating solution at $40^{\circ} \mathrm{C}$, and current density $1 \mathrm{~mA} / \mathrm{cm}^{2}$. Gold sulfite $\left[\mathrm{Au}\left(\mathrm{SO}_{3}\right)_{2}\right] \mathrm{Na}_{3}$ is the active ingredient and the solution operates at neutral $\mathrm{pH}$. From atomic force microscopy (AFM) measurements, a roughness of $11.88 \mathrm{~nm}(\mathrm{rms})$ was established to be typical for a 1-um-thick gold layer obtained with this solution. After the electroplating step the undisturbed flat backside of the patterned single crystal of $\mathrm{NbSe}_{2}$ was subsequently cleaved several times until the underlying etched/electroplated gold pattern was uncovered (Fig.1d and 1f). The latter procedure was performed in an inert atmosphere of He gas to avoid contamination of the freshly exposed $\mathrm{NbSe}_{2}$ surface. This 
method produces a clean atomically flat $\mathrm{NbSe}_{2}$ single crystal surface which is imbedded with laterally patterned gold islands. The sample was then immediately transferred into a low-temperature scanning tunnelling microscope and cooled down to $4.2 \mathrm{~K}$. This novel approach in fabricating patterned atomically flat single crystal surfaces free of surface contaminants is a key requirement for performing scanning tunnelling spectroscopy imaging of vortices (Fig. 1g)[4,5].

References

[1] T. Nishizaki, A. M. Troyanovski, G. J.C. van Baarle, P. H. Kes, and J. Aarts, Physica C 388, 777 (2003).

[2] G. J.C. van Baarle, A. M. Troyanovski, P. H. Kes, and J. Aarts, Physica C 369, 335 (2002).

[3] C. S. Oglesby, E. Bucher, C. Kloc, and H. Hohl, J. Cryst. Growth 137, 289 (1994).

[4] G. Karapetrov et al., Phys. Rev. Lett. 95, 167002 (2005).

[5] The authors would like to acknowledge M. Moldovan (Northwestern University) and D.

Rosenmann (Argonne) for their help with sample preparation and V. Vlasko-Vlasov (Argonne) and M. Zalalutdinov (Cornell) for useful discussions. Part of this work was carried out at the Center for Microanalysis of Materials, University of Illinois at Urbana-Champaign, which is partially supported by the U.S. Department of Energy under grant DEFG02-91-ER45439. This work has been supported by the U.S. DOE, BES-Material Sciences under contract No. W-31-109-ENG-38.
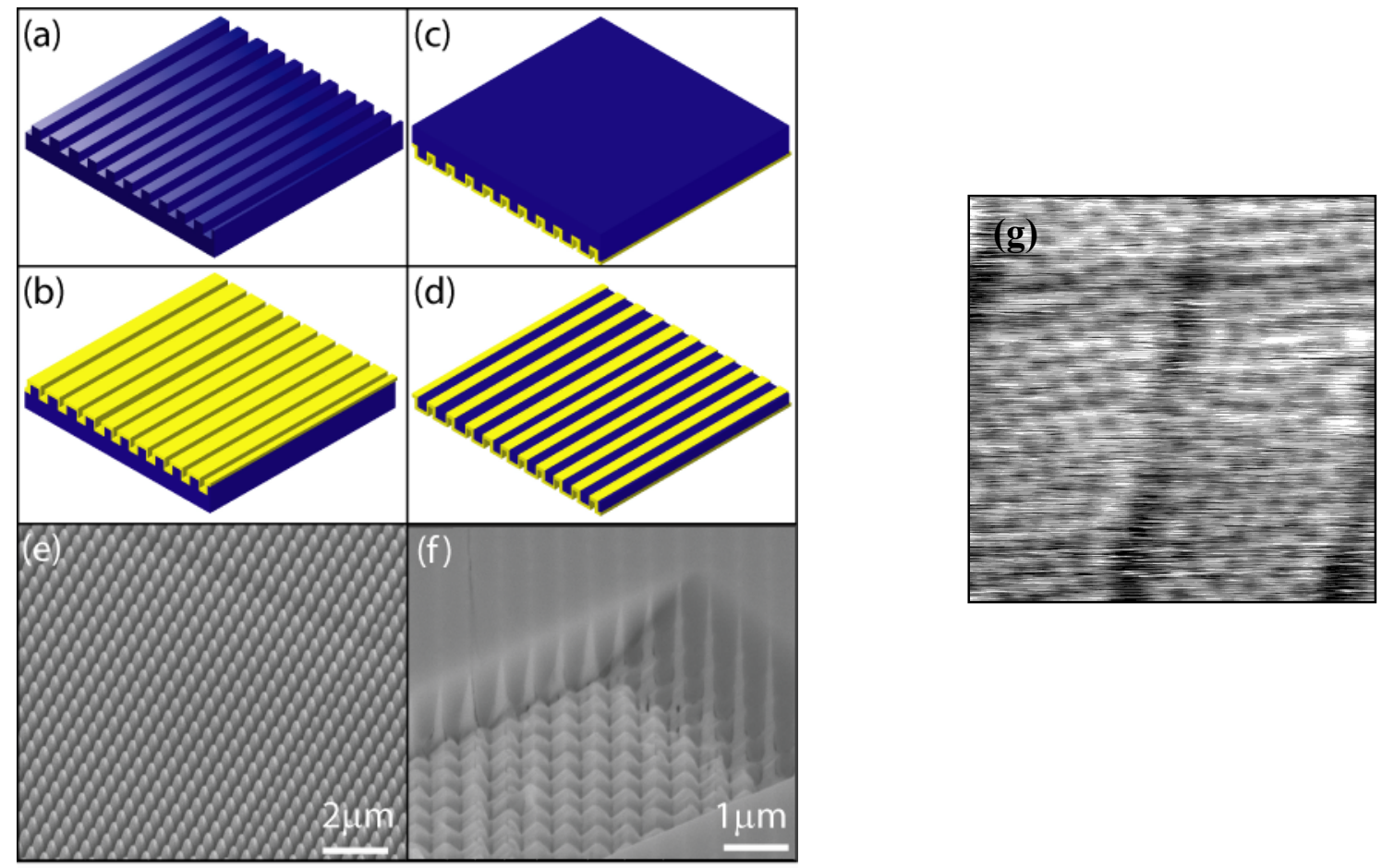

Fig. 1. Microfabrication of sub-micron mesoscopic single crystal superconductors using high aspect ration FIB patterning (a) is followed by gold electroplating (b) with subsequent backside exposure (c) and cleaving (d). SEM micrographs show the $\mathrm{NbSe}_{2}$ single crystal surface after performing FIB patterning with periodic structure containing a square array of pillars (e) and the surface and crosssectional structure after the final cleavage step (f). At the bottom of the FIB milled box one can see the electroplated gold that penetrates the interstitials of the single crystal. STM image $(1.25 \mu \mathrm{m} x$ $1.25 \mu \mathrm{m})$ of the vortex lattice at $4.2 \mathrm{~K}$ in magnetic field of $0.6 \mathrm{~T}(\mathrm{~g})$. 